\title{
Estimated glomerular filtration rate and drug dose adjustment in patients in an intensive care unit in Brazil
}

\author{
Marianne Silveira Camargo ${ }^{*}$, Márcio Galvão Oliveira ${ }^{2}$, \\ Sostenes Mistro ${ }^{\oplus 3}$, Izabel Cristina Pereira Rocha ${ }^{4}$, Camila Neto Santos ${ }^{5}$, \\ Caroline Tianeze de Castro ${ }^{6}$, Luiz Carlos Santana Passos ${ }^{7}$ \\ ${ }^{1}$ Universidade Federal da Bahia, Instituto Multidisciplinar em Saúde, Programa de Residência, \\ Salvador, Brazil, 2,3 Universidade Federal da Bahia, Instituto Multidisciplinar em Saúde, Programa \\ de Pós-Graduação em Saúde Coletiva, Vitória da Conquista, Brazil, ${ }^{4,5,6}$ Universidade Federal \\ da Bahia, Instituto Multidisciplinar em Saúde, Vitória da Conquista, Brazil, ${ }^{7}$ Universidade \\ Federal da Bahia, Programa de Pós-Graduação em Medicina e Saúde, Salvador, Brazil
}

\begin{abstract}
Objectives. This study sought to compare the estimated glomerular filtration rate and the indication of dose adjustment of antimicrobials when using Cockcroft-Gault or Modification of Diet in Renal Disease. Methods. A cross-sectional study was performed with patients admitted to the intensive care unit of a Brazilian general hospital. The glomerular filtration rate was calculated for patients on all days using the Cockcroft-Gault and Modification of Diet in Renal Disease equations. The difference in estimated glomerular filtration and the dose adjustment indication of antimicrobials were assessed. Results. A total of 631 patients were included in this study. The median estimated glomerular filtration was significantly higher when estimated using Modification of Diet in Renal Disease $(100.3 \mathrm{~mL} /$ $\left.\mathrm{min} / 1.73 \mathrm{~m}^{2}\right)$ than the estimation by Cockcroft-Gault $(83.2 \mathrm{~mL} / \mathrm{min})[\mathrm{p}<0.001]$. Greater differences in estimations produced by the two formulae were observed in patients at extremes of weight and age, and a different dose adjustment was indicated for all antimicrobials assessed. Conclusions. These results demonstrate a significant difference in estimated glomerular filtration rate values when calculated using either Cockcroft-Gault or Modification of Diet in Renal Disease as well as in the indication of dose adjustment in an intensive care unit.
\end{abstract}

Keywords: Intensive Care Units. Glomerular Filtration Rate. Anti-infective Agents. Renal Insufficiency.

\section{INTRODUCTION}

Kidney disease affects approximately 850,000 people annually around the world and represents the 12th leading cause of mortality in the general population (WHO, 2018). Patients affected by loss of renal function present with a high risk of adverse drug events, particularly due to the changes in the pharmacokinetics of numerous renally excreted substances. This results in

*Correspondence: M. Camargo, Universidade Federal da Bahia, Rua Padre Feijó, S/N, Canela, $5^{\circ}$ andar, Salvador 40110-060 - Brazil. E-mail: marianne_camargo@hotmail.com a need to adjust dosages with the aim of administering an optimal dose and reducing the risk of toxicity. In addition, drug overdose prescriptions have often been associated with the loss of renal function since many medications, including antihypertensive drugs, antimicrobials and analgesics, are contraindicated or require special care in cases such as these (KarschVolk et al., 2013; Nielsen et al., 2014). These drugs are widely used in the intensive care unit, where loss of renal function occurs in higher incidence (Cardinal et al., 2012; Uchino et al., 2005).

Acute kidney injury (AKI) is a very common condition in ICU patients and is associated with 
mortality rates of $50-60 \%$, despite the advances of medical care (Tejera et al., 2017). Therefore, accurately assessing the kidney function in critically ill patients is pivotal to reduce the severity of complications by supportive therapy as stabilization of the hemodynamic status, prompt treatment of any underlying disease and carefully assessment of nephrotoxic drugs use (Bragadottir, Redfors, Ricksten, 2013; Macedo, Mehta, 2013). In addition, it is essential for the appropriate dosing of drugs aiming to avoid toxicity caused by overdose (Pazhayattil, Shirali, 2014).

The glomerular filtration rate (GFR) is considered the best marker for monitoring renal function throughout its diagnosis, staging, and progression (Katsube et al., 2017). It can be precisely measured by markers such as iohexol, inulin, iodine-125 iothalamate, Cr-51 ethylenediaminetetraacetic acid (Cr-51 EDTA), and Tc-99m diethylenetriaminepenta-acetic acid (Tc-99m DTPA). However, these tests are expensive, laboriousness, and generally unavailable in routine hospital settings (Martin et al., 2011). Given these drawbacks to calculating GFR, equations such as the Modification of Diet in Renal Disease (MDRD) and Cockcroft-Gault (CG), which estimate GFR and creatinine clearance level, respectively, have been developed and used for this purpose (Cockcroft, Gault, 1976; Levey et al., 2006). However, although widely employed, these two equations are limited in their use (Helou, 2010).

The CG equation is based in data from a study conducted in 1973 that included 236 patients aging from 18 to 92 years, with mean weight of $72 \mathrm{~kg}$. From that total, only 10 patients were females. The CG equation includes age, sex, total body weight and serum creatinine (Cockcroft, Gault, 1976). On the other hand, MDRD is based on a study including patients with chronic renal disease, which assessed the clearance of iotalamato-I125. The brief form of MDRD includes the variables age, sex, ethnicity and serum creatinine and in the complete form, two other variables are included: serum albumin and blood urea nitrogen (BUN) (Levey et al., 1999; Levey et al., 2006).

Using the creatinine as a marker of renal function have some limitation since it is influenced by several factors as muscle mass, protein intake, physical activity or the use of medicines. In addition, the creatinine is excreted at a variable rate that increases as the renal failure progresses. Therefore, the clearance of creatinine overestimates the real value of the glomerular filtration rate (Kumar, Mohan, 2017).
Given the potential complications and risks associated with the avoidance of drugs during reduced GFR, several studies have addressed this subject. However, despite the higher incidence of renal dysfunction and the lack of standards in dose adjustments during ICU admission, there are few studies that have assessed the appropriateness of the equations and drug doses in patients with RF under intensive care.

This study sought to compare the estimated GFR (eGFR) and the recommendations for dose adjustment of broad-spectrum antimicrobials using $\mathrm{CG}$ and 4-Variable MDRD as well as the differences according to age and weight.

\section{METHODS}

This was a cross-sectional study, performed in a general hospital located in state of Bahia, in Brazilian Northeast. This institution is classified as a mediumand high-complexity urgent and emergency hospital that serves 134 municipalities with a total of 254 beds (Bahia, 2015). Data were collected from medical records using a form developed in the KoBoToolbox for Android (available at: www.kobotoolbox.org).

All patients admitted between January 2014 and December 2015 who were older than 18 years of age were included in this study. Patients who stayed in the ICU for less than 24 hours, who underwent dialysis or another renal replacement therapy, or whose missing data did not allow for the calculation of eGFR were excluded from this study.

All information contained in the medical records of patients was monitored and analysed from the day before ICU admission to the last day of hospitalization in ICU. The following data were collected from patients records: cause of admission, previous location of the patient before UCI admission (source), weight, height and serum creatinine. Then, these data were transferred to a database for further analysis.

The eGFR was calculated for each day of hospitalization in the ICU, using online calculators from the Brazilian Nephrology Society website (available at: https://sbn.org.br/utilidades/calculadoras/) and double checked using the following formulae:

CG: [(140 - age $) \times$ weight/(72 x serum creatinine $)] \mathrm{x}$ 0.85 (if female)(Cockcroft, Gault, 1976). 
MDRD: (186 x serum creatinine)- $1.154 \mathrm{x}$ age- 0.203 ) x 0.742 (if female) x 1.21 (if African American) (Levey et al., 2006).

Additionally to the comparison between the GFR estimated by CG and MDRD-4, we assessed the difference for dose adjustment recommendations when using the CG or MDRD-4 equations. Therefore, we choose the top five intravenous broad-spectrum antimicrobials requiring dose adjustments in patients with reduced glomerular filtration rate, used in our institution: vancomycin, meropenem, piperacillin/ tazobactam, cefepime, and fluconazole. The need for dose adjustment was assessed based on the following sources of information: Micromedex Healthcare Series ${ }^{\circledR}$ and AHFS - Drug Information Handbook 2015 (Micromedex, 2016; APhA, 2015).

Data were analysed with SPSS 23.0. Continuous variables are presented as the median and corresponding measure of dispersion. Proportions were compared using Pearson's chi-squared test. We compared median values between groups using the Wilcoxon signed-rank test, with a significance of $5 \%$.

Ethical: The study was approved by the Research Ethics Committee at Multidisciplinary Institute of Health, Federal University of Bahia, Vitória da Conquista, Brazil, with number: 1.460.914

\section{RESULTS}

The study included 631 patients. Table I describes the clinical and demographic characteristics of the population.

Most patients were male $(64.2 \%)$ with a median weight of $69.3 \mathrm{~kg}$. Admissions were most frequently due to neurological $(34.6 \%)$ and surgical $(32.3 \%)$ causes.

Median eGFR was $100.3 \mathrm{~mL} / \mathrm{min} / 1.73 \mathrm{~m}^{2}$ for MDRD-4 and $83.2 \mathrm{~mL} / \mathrm{min}$ for $\mathrm{CG}(\mathrm{p}<0.001)$.

Due to differences in eGFR results calculated using the CG or MDRD, there may be differences in the indication of drug dose adjustment. In table II, for each chosen antimicrobial, is compared the number of times the dose adjustment should be conducted according to the respective eGFR range, following the recommended dose adjustment, when the GFR is estimated by CG and MDRD-4 equations.

The study showed a greater difference in eGFR between equations in patients at extremes of weight and age. Figures 1 and 2 show the relationship between median eGFR, as determined using CG and MDRD-4 versus weight and age, respectively.

Table I - Demographic characteristics of the study population

\begin{tabular}{lc}
\hline Characteristics & (N=631) \\
\hline Sex & $\mathbf{n}(\mathbf{\%})$ \\
Male & $405(64.2)$ \\
Female & $226(35.8)$ \\
\hline
\end{tabular}

Source

Emergency

$349(55.3)$

Surgery centre

Surgical clinic

Other hospital

Medical clinic

$28(4.4)$

Other

Reason for hospitalization

(by organ system)

Neurologic

$218(34.6)$

Surgical

$204(32.3)$

Pulmonary

Cardiac

Gastrointestinal

Endocrinal

$10(1.6)$

Drug ingestion and overdose

Others

$109(17.3)$

Median (IQR*)

Age (years)

$50(32-67)$

Weight (kg)

$70(60-75)$

Baseline serum creatinine

$1(0.7-1.9)$

Length of stay in Intensive Care Unit

$6(3-12)$

*Interquartile range 
Table II - Frequency of dose adjustment according to recommended for each estimated glomerular filtration range calculated using CG or MDRD for broad-spectrum antimicrobials $(\mathrm{N}=5,528)$

\begin{tabular}{|c|c|c|c|c|}
\hline Drug & $\begin{array}{c}\text { Creatinine } \\
\text { clearance range }\end{array}$ & $\begin{array}{c}\text { eGFR calculated } \\
\text { using CG }(\%)\end{array}$ & $\begin{array}{l}\text { eGFR calculated } \\
\text { using MDRD (\%) }\end{array}$ & p-value* \\
\hline \multirow{2}{*}{ Vancomycin } & $10-50$ & $1587(28.7)$ & $1377(24.9)$ & \multirow{2}{*}{$\mathrm{p}<0.001$} \\
\hline & $<10$ & $65(1.2)$ & $78(1.41)$ & \\
\hline \multirow{3}{*}{ Meropenem } & $25-50$ & $891(16.1)$ & $797(14.4)$ & \multirow{3}{*}{$\mathrm{p}<0.001$} \\
\hline & $10-25$ & $696(12.6)$ & $580(10.5)$ & \\
\hline & $<10$ & $65(1.2)$ & $78(1.4)$ & \\
\hline \multirow{3}{*}{ Cefepime } & $30-60$ & $1062(19.2)$ & $854(15.4)$ & \multirow{3}{*}{$\mathrm{p}<0.001$} \\
\hline & $11-30$ & $832(15)$ & $729(13.2)$ & \\
\hline & $<11$ & $88(1.6)$ & $103(1.9)$ & \\
\hline \multirow{2}{*}{ Piperacillin/Tazobactam } & $20-40$ & $849(15.4)$ & $741(13.4)$ & \multirow{2}{*}{$\mathrm{p}<0.001$} \\
\hline & $<20$ & $473(8.6)$ & $408(7.4)$ & \\
\hline Fluconazole & $<50$ & $1652(19.9)$ & $1455(26.3)$ & $\mathrm{p}<0.001$ \\
\hline
\end{tabular}

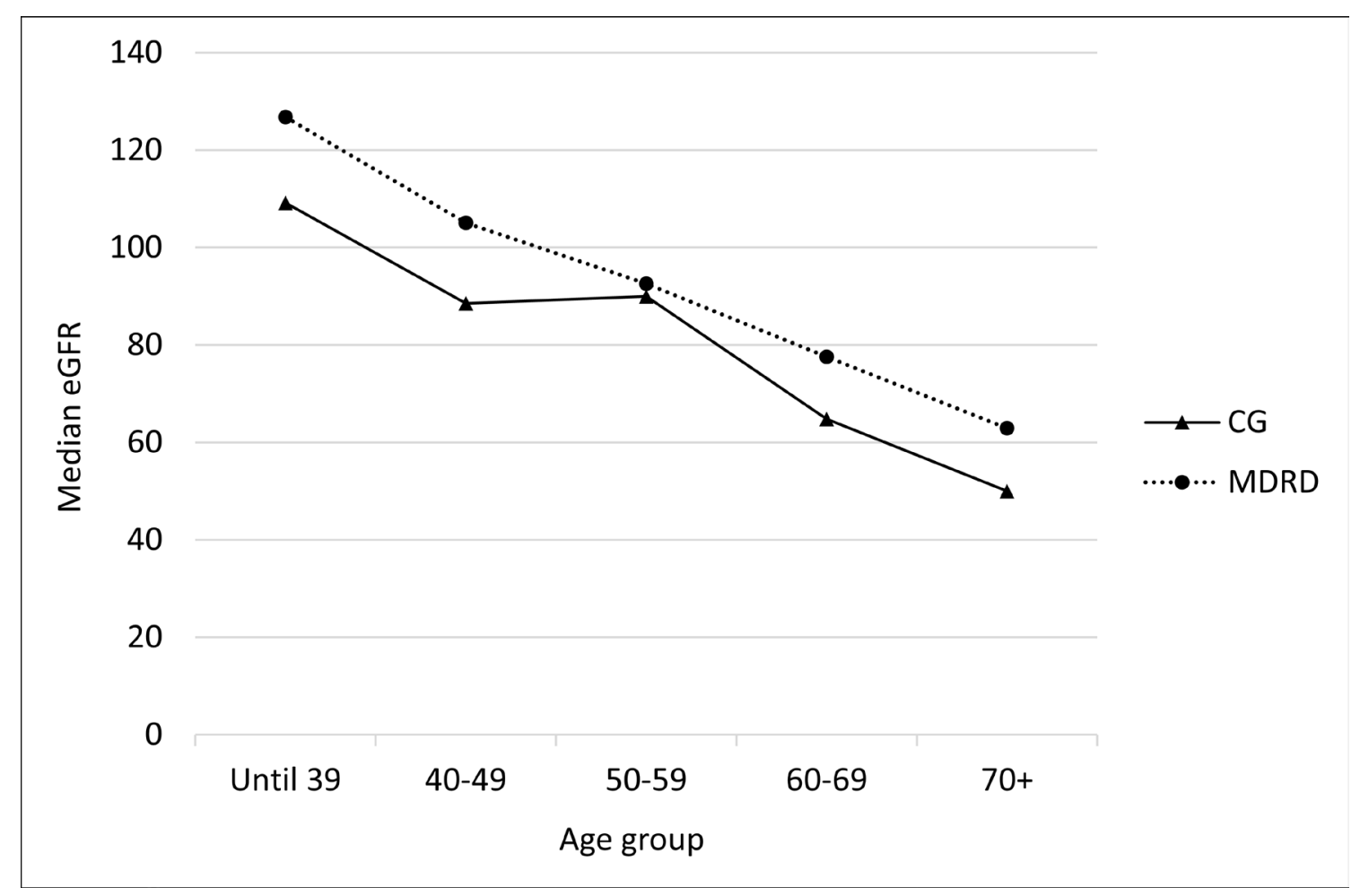

Figure 1 - Median estimated glomerular filtration rate calculated using MDRD-4 or Cockcroft-Gault versus age group. 


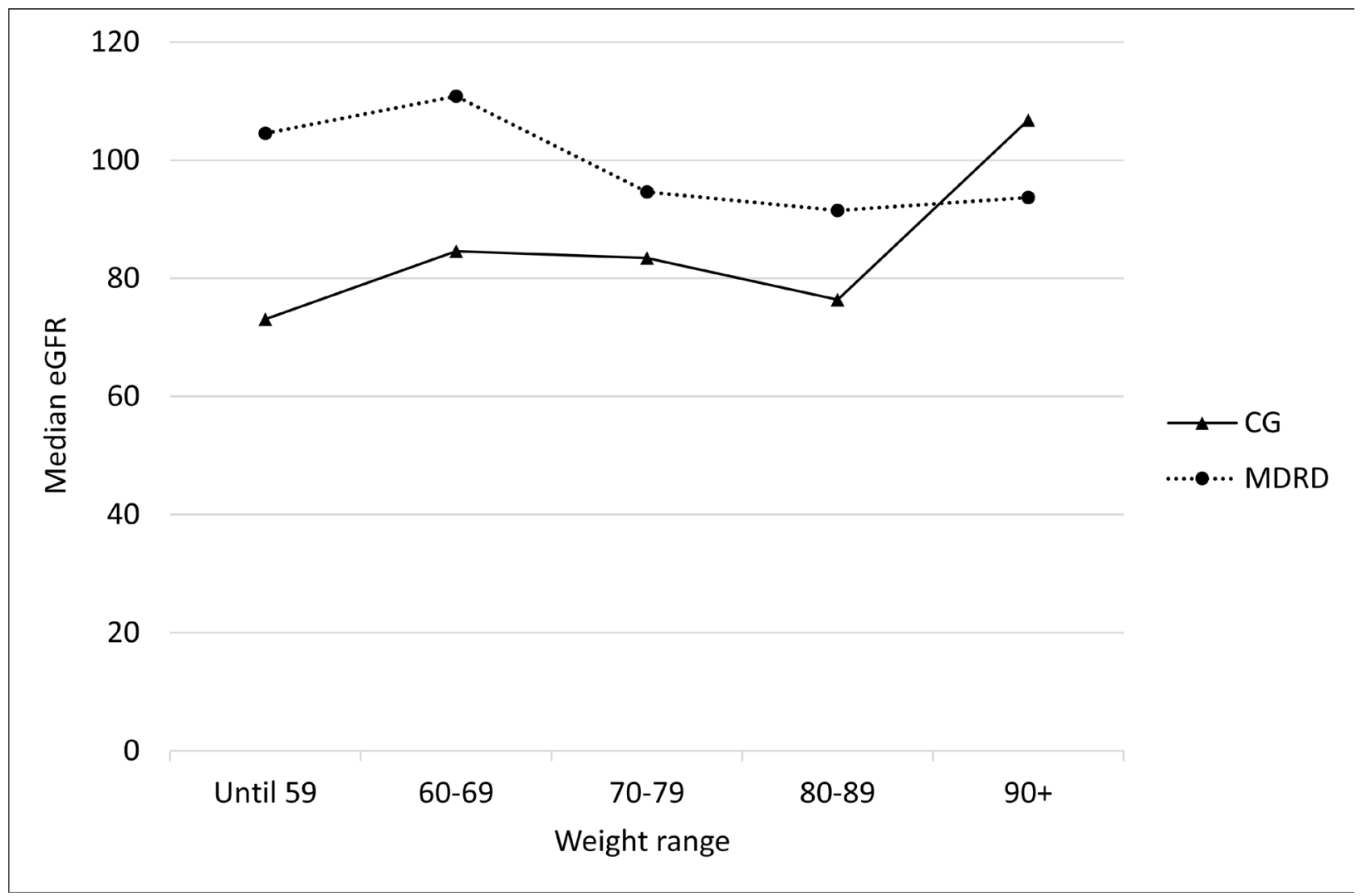

Figure 2 - Median estimated glomerular filtration rate calculated using MDRD-4 or Cockcroft-Gault versus weight range.

\section{DISCUSSION}

This study demonstrated a significant difference in eGFR in ICU patients when calculated using MDRD4 and CG. Disagreements between the equations raise doubts about how the dose adjustment should be performed, which can lead to serious errors, including medication errors. To reduce this difference between the equations, eGFR can be calculated using the body surface area (BSA), although this data is difficult to obtain in medical records. Furthermore, in many cases, its measurement is imprecise. In addition, the impact of BSA correction seems to be limited (Roblin et al., 2009).

As observed in Figure 1, in the age group of 50 to 59 years, eGFR values are similar, although the difference becomes more significant with advanced age. This difference between eGFR, as calculated by either CG or MDRD-4, in elderly patients has been previously observed. It was found that GFR, when compared to the gold standard, was underestimated when calculated using CG and overestimated when calculated using MDRD (Saha et al., 2015). Similarly, a study showed a difference in dose adjustment between the equations for drugs, such as amantadine and digoxin, which are commonly used in elderly patients (Gill et al., 2007). In this population, it is common to find patients with reduced muscle mass and, consequently, very low serum creatinine levels. Some studies suggest rounding these creatinine levels to $1 \mathrm{mg} / \mathrm{dL}$, although this practice may lead to an underestimation of creatinine clearance levels. Thus, some studies continue to suggest using CG as a safer option for these patients since use of MDRD-4 results in a higher estimation of GFR, which may result in higher adjusted doses.

In this study, the difference observed between eGFR calculated using either CG or MDRD-4 was also significantly increased at extremes of weight, as seen in Figure 1. As expected, up to the weight range of 70$79 \mathrm{~kg}$, eGFR as calculated using CG is lower than that 
calculated using MDRD-4. This is because only the CG equation considers weight, which is a positive factor in this equation. Conversely, GFR tends to be higher in obese patients than in the rest of the population, which causes a greater discrepancy in GFR and more dramatically affects the adjustment of drug dosage (Jesudason, Clifton, 2012; Nyman et al., 2011).

In our study, we used the total body weight (TBW) of the patients, since this is the recommended weight used in the original study that defined the CG equation (Cockcroft, Gault, 1976). Some authors state that the use of ideal body weight (IBW) is indicated for patients at the extremes of weight, although, in these cases, an underestimation of GFR may occur (Hudson, Mason, Huch, 2011). Other authors indicate the use of a cofactor to increase the reliability of the CG equation (Wilhelm, Kale-Pradhan, 2011). Therefore, prior to drug dose adjustment, it is important to seek guidance from the pharmaceutical company that produces the drug to determine whether the recommendation for dose adjustment is made based on TBW or IBW. In some cases, this information can be found in the package leaflet (Nyman et al., 2011).

In patients at extremes of weight and age, who showed higher eGFR difference between the equations, the results suggest a need for greater care in the application of the equations. Many studies have been conducted with specific groups and have compared them with the gold standard, although there is no clear answer as to the best form and equation to calculate eGFR.

A feature similar to both equations used to calculate eGFR is the dependence on serum creatinine. Both serum creatinine and BUN are often excreted by kidneys in a constant way and therefore are commonly used as markers to assess renal function. Although, since several factors such as muscle mass, diet, hydration, analytical variations or even ethnicity can influence the excretion of these two markers, their use in some groups may be not safe for eGFR (Kumar, Mohan, 2017). The Brazilian population is the result of an intense process of miscegenation between several different ethnicities, including Europeans, black Africans, and indigenous people, which resulted from colonization and the intense immigration that occurred in recent centuries (Brasil, 2009). Thus, unlike previous data from groups with low ethnic variability, the results of this study were obtained from a widely multiracial population. Likewise, this study included ICU patients who are frequently affected by hemodynamic instability, which other studies have found to be an uncommon condition (Potes et al., 2017).

In order to assess the patient throughout the hospitalization period and to consider all variability that occurs during that period, such as weight changes, diet, and use of medications, the GFR was estimated daily using both equations and all test results were classified according to the creatinine clearance range used to indicate the dose adjustment (Table II). In agreement with previous data (Golik, Lawrence, 2008; Kumar, Mohan, 2017), this study demonstrated that different suggestions of dose adjustment could occur according to the equation used for eGFR. For all creatinine clearance ranges, significant differences were found between test results. These results indicate that deciding which dose should be prescribed, according to eGFR alone, can be harmful to the patient, since both a sub-dose and overdose of medications can be harmful (Brown, Masselink, Lalla, 2013).

Several broad-spectrum antibiotics, commonly used in ICU, are potentially nephrotoxic while are mainly excreted by the kidneys. Vancomycin, for example, is widely used for the treatment of infections caused by resistant strains of gram-positive bacteria but presents high nephrotoxicity rates (Luque, Mesnard, 2018; Park et al., 2018). Other agents as piperacillin/ tazobactam, meropenem, imipenem and cefepime, active against gram-negative bacteria, are associated with lower nephrotoxic rates but may cause central nervous toxicity in overdose or can to potentiate the toxicity of other antibiotics such as vancomycin. Hence, using those broad-spectrum antibiotics require close attention in renal function aiming to avoid its depletion as well as to prevent kidneys or other organ injury caused by overdoses of these drugs resulting from loss of glomerular filtration (Cook et al., 2018).

Given the risks inherent to dose adjustment of certain drugs, it is necessary to consider which decision would carry reduced risk to the patient when there is a difference in the indication of dose adjustment. For example, in addition to higher costs, a higher than necessary dose carries a risk of adverse reactions, such as convulsion and myoclonus. Conversely, if the dose is lower than necessary, therapeutic failure may occur, thereby leading to an increased length of stay, a worsening of clinical status, and even death (Hudson, Mason, Huch, 2011; Nyman et al., 2011). Moreover, eGFR is also used to guide nutritional support and the 
need for renal replacement therapy (Brown, Masselink, Lalla, 2013).

In 1998, the Food and Drug Administration (FDA) published guidelines for the pharmaceutical industry, which suggests the use of the CG equation to estimate renal function since it was the most commonly used equation for this purpose (FDA, 1998). In 2010, the FDA published new guidelines recommending the use of both the creatinine clearance level and GFR for drug dose adjustment, which makes it possible to use the MDRD-4 equation (FDA, 2010). However, for previously approved drugs, it is unlikely that any changes will be made, since tests have already been performed and these drugs are already on the market. Thus, for some drugs that have dose adjustment instructions based on the CG equation, the use of MDRD-4 may have important implications since this equation usually suggests higher doses than does $\mathrm{CG}$, which results in a greater uncertainty in their implementation (Golik, Lawrence, 2008; Hermsen et al., 2009).

The difficulty in estimating GFR in ICU patients is associated with several factors, such as sudden changes in renal volume and haemodynamic activity, which are common in this population. In individuals with frequent changes in serum creatinine concentration or with acute kidney injury, the use of equations should be performed with caution since none are considered safe in these cases and have not been completely validated for this purpose (Cockcroft, Gault, 1976; Levey et al., 2006; Sunder et al., 2014). Clinicians often suggest assessing urine for various markers over a period of 24 hours to estimate GFR. However, this method is not precise and requires additional resources and time to obtain results (El-Minshawy, Saber, Osman, 2010).

Choosing one over another between CG and MDRD may lead to the prescription of a medication overdose or to the treatment switch, based on the eGFR result. Various formulae were created after CG and considered better than it. However, there is no consensus on which one is better to estimate the GFR. Thus, the choice must take into consideration patients' individual characteristics and the drug therapy aim, regarding risks and benefits (Cartet-Farnier et al., 2017; Delanaye et al., 2017).

We did not include in the comparison other equations to estimate GFR as the CKD-EPI Creatinine Equation. However, our results cover the majority of GFR estimations conducted in our settings, especially for doses adjustments, since CG and MDRD are most frequently applied. Moreover, we did not compare the
eGFR by CG and MDRD with the creatinine clearance rate measured by gold-standards markers as iohexol or inulin because there are not often used in a routine basis.

This study demonstrated a significant difference in eGFR values when calculated using either CG or MDRD-4 as well as in the indication of dose adjustment of broad-spectrum antimicrobials administered to patients admitted in an ICU. In the analysis, weight and age have influenced for higher divergence between the eGFR obtained by the two equations.

\section{REFERENCES}

Micromedex 2.0. Truven Health Analytics, Inc. Greenwood Village, CO. [Internet]. 2016. Disponívem em: http://www. micromedexsolutions.com.

APhA. Drug Information Handbook: Lexicomp; 2015. 2260 p.

Bahia. Secretaria de Saúde do Estado da Bahia. Estudo Unidades Hospitalares [internet]. Bahia (Ba): Secretaria de Saúde, 2015. [Citado 2017 Jan 7]. Disponível em: http://www. saude.ba.gov.br/.

Bragadottir G, Redfors B, Ricksten SE. Assessing glomerular filtration rate (GFR) in critically ill patients with acute kidney injury--true GFR versus urinary creatinine clearance and estimating equations. Crit Care. 2013;17(3):R108.

Brasil. Governo e Política. População 2009 [cited 2016 December, 12]. Available from: http://www.brasil.gov.br/ governo/2009/11/populacao.

Brown DL, Masselink AJ, Lalla CD. Functional range of creatinine clearance for renal drug dosing: a practical solution to the controversy of which weight to use in the CockcroftGault equation. Ann Pharmacother. 2013;47(7-8):1039-44.

Cartet-Farnier E, Goutelle-Audibert L, Maire P, De la Gastine B, Goutelle S. Implications of using the MDRD or CKD-EPI equation instead of the Cockcroft-Gault equation for estimating renal function and drug dosage adjustment in elderly patients. Fundam Clin Pharmacol. 2017;31(1):110-9.

Cockcroft DW, Gault MH. Prediction of creatinine clearance from serum creatinine. Nephron. 1976;16(1):31-41.

Cook KM, Gillon J, Grisso AG, Banerjee R, Jimenez-Truque $\mathrm{N}$, Phillips EJ, et al. Incidence of Nephrotoxicity Among Pediatric Patients Receiving Vancomycin With Either Piperacillin-Tazobactam or Cefepime: A Cohort Study. J Pediatric Infect Dis Soc. 2018.

Delanaye P, Guerber F, Scheen A, Ellam T, Bouquegneau A, Guergour D, et al. Discrepancies between the Cockcroft- 
Gault and Chronic Kidney Disease Epidemiology (CKD-EPI) Equations: Implications for Refining Drug Dosage Adjustment Strategies. Clin Pharmacokinet. 2017;56(2):193-205.

El-Minshawy O, Saber RA, Osman A. 24-hour creatinine clearance reliability for estimation of glomerular filtration rate in different stages of chronic kidney disease. Saudi J Kidney Dis Transpl. 2010;21(4):686-93.

Gill J, Malyuk R, Djurdjev O, Levin A. Use of GFR equations to adjust drug doses in an elderly multi-ethnic group--a cautionary tale. Nephrol Dial Transplant. 2007;22(10):2894-9.

Golik MV, Lawrence KR. Comparison of dosing recommendations for antimicrobial drugs based on two methods for assessing kidney function: cockcroft-gault and modification of diet in renal disease. Pharmacotherapy. 2008;28(9):1125-32.

Helou R. Should we continue to use the Cockcroft-Gault formula? Nephron Clin Pract. 2010;116(3):c172-85; discussion c86.

Hermsen ED, Maiefski M, Florescu MC, Qiu F, Rupp ME. Comparison of the Modification of Diet in Renal Disease and Cockcroft-Gault equations for dosing antimicrobials. Pharmacotherapy. 2009;29(6):649-55.

Hudson JQ, Mason DL, Huch KM. Estimates of kidney function in obese African Americans with chronic kidney disease. Nephron Clin Pract. 2011;118(2):c101-8.

Jesudason DR, Clifton P. Interpreting different measures of glomerular filtration rate in obesity and weight loss: pitfalls for the clinician. Int J Obes (Lond). 2012;36(11):1421-7.

Karsch-Volk M, Schmid E, Wagenpfeil S, Linde K, Heemann U, Schneider A. Kidney function and clinical recommendations of drug dose adjustment in geriatric patients. BMC Geriatr. 2013;13:92.

Katsube T, Wajima T, Ishibashi T, Arjona Ferreira JC, Echols R. Pharmacokinetic/Pharmacodynamic Modeling and Simulation of Cefiderocol, a Parenteral Siderophore Cephalosporin, for Dose Adjustment Based on Renal Function. Antimicrob Agents Chemother. 2017;61(1).

Kumar BV, Mohan T. Retrospective Comparison of Estimated GFR using 2006 MDRD, 2009 CKD-EPI and CockcroftGault with 24 Hour Urine Creatinine Clearance. J Clin Diagn Res. 2017;11(5):BC09-BC12.

Levey AS, Bosch JP, Lewis JB, Greene T, Rogers N, Roth D. A more accurate method to estimate glomerular filtration rate from serum creatinine: a new prediction equation.
Modification of Diet in Renal Disease Study Group. Ann Intern Med. 1999;130(6):461-70.

Levey AS, Coresh J, Greene T, Stevens LA, Zhang YL, Hendriksen $\mathrm{S}$, et al. Using standardized serum creatinine values in the modification of diet in renal disease study equation for estimating glomerular filtration rate. Ann Intern Med. 2006;145(4):247-54.

Luque Y, Mesnard L. [Vancomycin nephrotoxicity: Frequency and mechanistic aspects]. Nephrol Ther. 2018;14 Suppl 1:S133-S8.

Macedo E, Mehta RL. Measuring renal function in critically ill patients: tools and strategies for assessing glomerular filtration rate. Curr Opin Crit Care. 2013;19(6):560-6.

Martin JH, Fay MF, Udy A, Roberts J, Kirkpatrick C, Ungerer J, et al. Pitfalls of using estimations of glomerular filtration rate in an intensive care population. Intern Med J. 2011;41(7):537-43.

Nielsen AL, Henriksen DP, Marinakis C, Hellebek A, Birn $\mathrm{H}$, Nybo M, et al. Drug dosing in patients with renal insufficiency in a hospital setting using electronic prescribing and automated reporting of estimated glomerular filtration rate. Basic Clin Pharmacol Toxicol. 2014;114(5):407-13.

Nyman HA, Dowling TC, Hudson JQ, Peter WL, Joy MS, Nolin TD. Comparative evaluation of the Cockcroft-Gault Equation and the Modification of Diet in Renal Disease (MDRD) study equation for drug dosing: an opinion of the Nephrology Practice and Research Network of the American College of Clinical Pharmacy. Pharmacotherapy. 2011;31(11):1130-44.

Park SJ, Lim NR, Park HJ, Yang JW, Kim MJ, Kim K, et al. Evaluation of risk factors for vancomycin-induced nephrotoxicity. Int J Clin Pharm. 2018.

Pazhayattil GS, Shirali AC. Drug-induced impairment of renal function. Int J Nephrol Renovasc Dis. 2014;7:457-68.

Potes C, Conroy B, Xu-Wilson M, Newth C, Inwald D, Frassica J. A clinical prediction model to identify patients at high risk of hemodynamic instability in the pediatric intensive care unit. Crit Care. 2017;21(1):282.

Roblin I, De Sobarnitsky S, Basselin C, Vial F, Bard E, Dufrene I, et al. Estimated glomerular filtration rate for drug dose adjustment: Cockcroft and Gault or abbreviated MDRD equation? Clin Biochem. 2009;42(1-2):111-3.

Cardinal L, Matos V, Resende G, Toffoli-Kadri M. Characterization of drug prescriptions in an adult intensive care unit. Rev Bras Ter Intensiva. 2012;24(2):151-6. 
Saha TK, Bhattarai AM, Batra HS, Banerjee M, Misra P, Ambade V. Correlation of Microalbuminuria with Estimated GFR (eGFR) by Cockcroft-Gault and MDRD Formula in Type 2 Diabetics and Hypertensives. Indian J Clin Biochem. 2015;30(3):271-4.

Sunder S, Jayaraman R, Mahapatra HS, Sathi S, Ramanan V, Kanchi P, et al. Estimation of renal function in the intensive care unit: the covert concepts brought to light. J Intensive Care. 2014;2(1):31.

Tejera D, Varela F, Acosta D, Figueroa S, Benencio S, Verdaguer $\mathrm{C}$, et al. Epidemiology of acute kidney injury and chronic kidney disease in the intensive care unit. Rev Bras Ter Intensiva. 2017;29(4):444-52.

U.S. Food and Drug Administration. Guidance for industry: pharmacokinetics in patients with impaired renal function- study design, data analysis, and impact on dosing and labeling 1998 [cited 2016 December, 10]. Available from: http://www.fda.gov/downloads/Drugs/ GuidanceComplianceRegulatoryInformation/Guidances/ ucm072127.pdf.
U.S. Food and Drug Administration. Draft guidance for industry: pharmacokinetics in patient with impaired kidney function - study design DA, and impact on dosing and labeling 2010 [cited 2016 December, 10]. Available from: http://www.fda.gov/downloads/Drugs/ GuidanceComplianceRegulatoryInformation/Guidances/ UCM204959.pdf.

Uchino S, Kellum JA, Bellomo R, Doig GS, Morimatsu H, Morgera S, et al. Acute renal failure in critically ill patients: a multinational, multicenter study. JAMA. 2005;294(7):813-8.

WHO. Statistical Information System (WHOSIS) [cited 2018 05 march]. Available from: http://www.who.int/whosis/en/.

Wilhelm SM, Kale-Pradhan PB. Estimating creatinine clearance: a meta-analysis. Pharmacotherapy. 2011;31(7):65864.

Received for publication on $15^{\text {th }}$ August 2017 Accepted for publication on $01^{\text {st }}$ February 2019 